\title{
Acciones ante la pandemia COVID-19 para mantener la calidad docente en la UVic-UCC
}

\section{Eulàlia Massana-Molera $^{\mathrm{a}}$, Richard Samson ${ }^{\mathrm{b}}$, Elena Molina Vicente ${ }^{\mathrm{c}}$, Anna Ballús Pujol ${ }^{\mathrm{d}}$}

${ }^{A}$ Facultad de Empresa y Comunicación, y Unidad de Docencia Universitaria y Tecnología Educativa, Universidad de Vic - Universidad Central de Cataluña, eulalia.massana@uvic.cat, bFacultad de Educación, Traducción y Ciencias Humanas, y Unidad de Docencia Universitaria y Tecnología Educativa, Universidad de Vic - Universidad Central de Cataluña, rsamson@uvic.cat, 'Unidad de Docencia Universitaria y Tecnología Educativa, Universidad de Vic Universidad Central de Cataluña, elena.molina@uvic.cat y ${ }^{\mathrm{c} U n i d a d ~ d e ~ D o c e n c i a ~ U n i v e r s i t a r i a ~ y ~ T e c n o l o g i ́ a ~ E d u c a t i v a, ~}$ Universidad de Vic - Universidad Central de Cataluña, anna.ballus@uvic.cat

\begin{abstract}

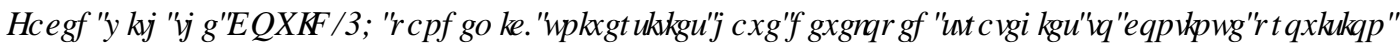

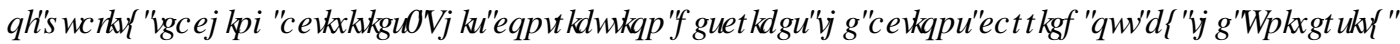

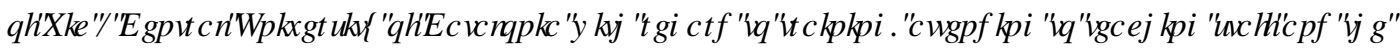

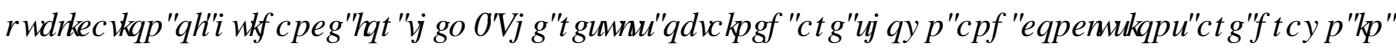
RUCHUTREXICFRQFXUHQHSLRJUHMII
\end{abstract}

ए

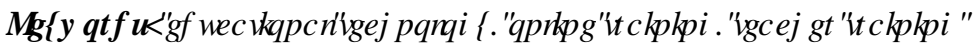

\section{Resumen}

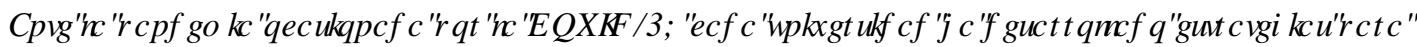

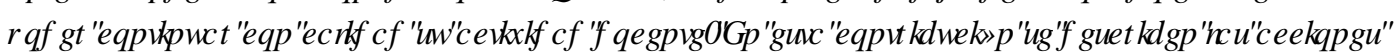

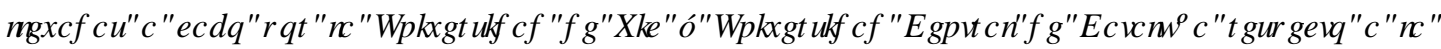

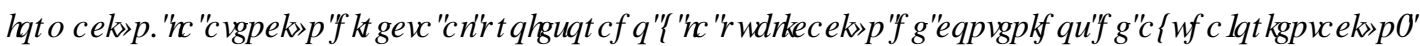

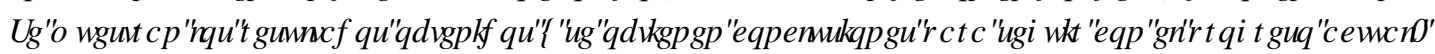

\section{DOEWVIFOYH}

\section{Introducción}

\subsection{Usos de las TIC en la docencia}

La transformación digital (TD) es el cambio hacia una mayor utilización de las Tecnologías de la Información y la Comunicación (TIC) en las organizaciones para aumentar su competitividad, sea porque su repercusión en los procesos los hace más rápidos, de más calidad, más económicos o porque nos permite acceder a mercados más amplios (Priesca, 2016; Crue, 2017).

Todos los aspectos de la sociedad actual están sometidos a esta TD, por lo que las universidades no son ajenas a esto. Y todos los procesos de las universidades están afectados por ella: desde la captación de estudiantes, la gestión, la investigación y la docencia, entre otros.

Entendemos como usos de las TIC en la docencia todas aquellas prácticas que usan estas herramientas para el proceso de enseñanza-aprendizaje, siendo más los usos que se aplican que las características de la herramienta las claves de los resultados de su implementación (Adell y Castañeda, 2010; Coll HWDO 2008; Esteve, 2009; Crue, 2018). 
Hay estudios que plantean la relación de las TIC con la docencia desde diferentes perspectivas (ÁreaMoreira, 2016; Coll, 2004; Coll, 2008; Cuen, 2013; Gisbert, 2017; Gros, 2016; Hart, 2018 y 2020; Mercader, 2017 y 2018; Zempoalteca, 2017). La tecnología no es ni sólo una herramienta que facilita ciertas prácticas docentes, ni sólo permite hacer lo que ya se hacía. Cuando el diseño educativo y las tecnologías se comunican, pueden emerger prácticas que no siempre se pueden predecir (Gros, 2016).

Estos estudios plantean la relación entre usos TIC y docencia desde qué herramientas se usan, en qué momentos, por parte de qué actores o qué acciones se median con ellas, entro otros aspectos.

\subsection{Profesorado universitario y TIC}

Mercader (2018) explica que el perfil del docente puede ser identificado a partir de diferentes aspectos (metodología, liderazgo, paradigma que utiliza... incluidos los que tienen relación con las TIC (predisposición al uso, competencia digital, integración en la práctica profesional...) y recoge cómo otros actores identifican diferentes colectivos según este aspecto.

Todos los autores consultados por Mercader (2018) consideran que la competencia digital docente es un aspecto relevante para el desarrollo adecuado de las tareas del profesorado en el aula.

El profesorado universitario, en general, es quién decide la estrategia y metodología que aplicará en cada asignatura, según su criterio. Algunos autores relacionan esta decisión con la etapa de la carrera académica de este profesorado (Mercader, 2018).

Así, aunque el uso de las TIC en la docencia es considerado por muchos autores como una oportunidad para innovar y mejorar, el profesorado puede sentirse presionado e incómodo, ofreciendo resistencia al cambio (Córica, 2020). Mientras la decisión de su uso quede en manos del docente, sin una acción decisiva de la institución para incentivarlo, no quedaría más que ofrecer formación y recursos e intentar convencer al profesorado de hacer su propia TD. Esto genera una situación desigual según las universidades, sus estrategias y los recursos de que disponen (Crue, 2018).

\subsection{La transformación digital en las universidades catalanas}

Todas las universidades catalanas consideran necesaria y trabajan activamente para la innovación docente como mejora continua de la tarea formativa. Dentro de esta innovación docente se considera los usos de las TIC como herramienta a tener en cuenta.

Desde sus Institutos de Ciencias de la Educación (ICE) y/u otras estructuras propias, cada universidad afronta desde diferentes perspectivas las metodologías con las cuales pueden dar soporte a las TIC, la formación necesaria del profesorado y alumnado, la categorización de recursos y el acceso a experiencias y/o materia de referencia. Uno de los retos más importantes es cómo integrar este cambio (necesariamente continuado) en el profesorado y cómo se valora esto en su carrera profesional.

Las cuestiones de las herramientas TIC, sus usos y aplicaciones es un ámbito en constante evolución (Adams Becker HWDQ2017; Alexander HWDQ2019; Brown HWDQ2020).

\subsection{Y con la pandemia, ¿qué nos ha pasado?}

Todo lo comentado en los apartados anteriores de esta Introducción es lo que podríamos haber afirmado para el período anterior a marzo de 2020. Pero el contexto de la formación universitaria (como tantos otros de la sociedad) se ha visto abruptamente condicionado por la pandemia de COVID-19 y los constantes ciclos de confinamiento a los que nos hemos visto sometidos.

El paso de una docencia totalmente presencial a en línea (con más o menos presencialidad) ha sido no planificado y hemos hecho, en muy poco tiempo y a la fuerza, cambios en la TD que, sin esta situación, hubiéramos tardado años en hacer. Se ha abierto un espacio para el uso de las TIC en la docencia, y lo que 
era una oportunidad se ha convertido en una necesidad. La capacidad de adaptación y adecuación son críticas para seguir manteniendo la misma calidad de la formación de nuestros estudiantes. Y esto ha significado, también, cambios en el uso de las TIC en la docencia (Crue, 2020; Hart, 2020).

En la Universidad de Vic - Universidad Central de Cataluña (UVic-UCC), se han llevado a cabo diferentes acciones para adaptarse a esta situación. En este trabajo se describen estas acciones, sus resultados y las conclusiones a las que nos llevan éstos.

\section{Objetivos}

- Describir las acciones y los recursos aplicados en la UVic-UCC en el período de 11 de marzo del 2020 a 31 de enero de 2021, como consecuencia de los diferentes momentos y grados de confinamiento producidos por la pandemia COVID-19.

- Analizar el seguimiento y resultado de las acciones anteriormente descritas.

- Identificar cuáles son las mejores prácticas aplicadas.

- Establecer / reforzar acciones para hacer frente a una situación futura que se percibe incierta.

\section{Desarrollo de la innovación}

\section{1. ¿Qué es la UDUTE?}

La Unidad de Docencia Universitaria y Tecnología Educativa (UDUTE) de la UVIc-UCC nació como Unidad de Apoyo a la Docencia (USD) el año 2013, como instrumento de nexo y gestión para facilitar la adopción y el aprovechamiento de la plataforma de aprendizaje Moodle, en sustitución de un sistema propio anterior.

Desde sus inicios la USD trabajó para forjar alianzas dinámicas entre las áreas estancas tradicionales de la universidad: de un lado servicios, como el de informática, y del otro los departamentos responsables de la docencia y la investigación. Con recursos limitados la USD trazó una trayectoria de tercer espacio (Whitchurch, 2012) dentro de la universidad, creando equipos multidisciplinares para tender puentes entre personal técnico y académico. Esta manera de plantear la mejora docente a través del uso creciente de tecnología educativa siguió con una implantación creciente, pero con resistencias también, hasta la llegada de la pandemia.

La UVic-CC dispone de un modelo de formación que contempla el uso de la TIC como herramienta transversal, además de la diversificación de metodologías docentes y de evaluación. Antes de la pandemia, la adopción de nuevas estrategias fue desigual entre el profesorado, con mucho envío de archivos de documentos docentes y trabajos de estudiantes no interactivos ni audiovisuales, y con preponderancia de sesiones de clase en formato tradicional (expositivas por parte del profesorado), evaluación presencial (con gran peso de exámenes finales), entre otros.

En cambio, en el caso de cursos con presencialidad reducida o totalmente a distancia, los cambios en la metodología y el uso de las TIC ya había avanzado (sobre todo por necesidad) antes de marzo del 2020.

\section{2. ¿Qué hemos hecho?}

Desde el inicio del confinamiento, la USD (actualmente UDUTE), se organizó para dar apoyo y acompañamiento al personal de la UVic-UCC (especialmente al profesorado), ampliando y diversificando sus acciones.

Identificamos dos períodos principales: el primero, des del 11 de marzo a julio de 2020, y el segundo, desde el 1 de septiembre de 2020 hasta el 31 de enero de 2021 (fecha de recogida de datos para la redacción de esta comunicación). En el primer período la USD recibió un soporte extra parcial y en segundo, la unidad

(c)) BY-NC-ND 2021, Universitat Politècnica de València

CRQJUHR, Q5 HGHपिए। 
se reorganizó, convirtiéndose en la UDUTE, y se vio reforzada con nuevas incorporaciones (pasando de dos personas a cuatro, aunque no a dedicación completa).

Pocos días antes del inicio del primer confinamiento, la USD empezó a preparar lo que se preveía como una interrupción de unos pocos días de no presencialidad. Todas las actividades pasaron a ser en línea y las herramientas que se propusieron al profesorado fueron MS Teams (integrado en la licencia de MS 365 Education de la universidad) y Zoom para sesiones de grupos grandes.

Se planteó una estrategia de urgencia:

- Elaboración de material básico e indicaciones iniciales para orientar al profesorado en cómo adaptar una planificación docente totalmente presencial a totalmente en línea. Se publicaron diversos materiales de apoyo en el blog de la USD (mon.uvic.cat/udute).

- Puesta en marcha de URespon de la USD, un portal de peticiones ya usado por el Área de las TIC, adaptado a la unidad para que el profesorado pudiera pedir ayuda puntual.

- Edición de material (entradas de blog y videotutoriales) para usos concretos de herramientas ya disponibles y para el conocimiento de las nuevas herramientas.

- Sesiones en línea de soporte abiertas a todo el profesorado, algunas con temas concretos y otras abiertas a las inquietudes de los asistentes.

El confinamiento se alargó hasta final de semestre (evaluación incluida), con lo que se tuvieron que poner en marcha unas acciones complementarias:

- Gestión de reservas de sesiones de videoconferencia (que aumentaron considerablemente) haciendo uso de licencias comunitarias, descargas de las grabaciones y registro de la asistencia en estas sesiones. El hecho de compartir licencias hizo imprescindible una gestión administrativa para evitar conflictos y asegurar la disponibilidad de recursos.

- Descarga, edición y publicación de las grabaciones de las sesiones de formación.

- Asesoramiento en el uso de las nuevas herramientas de trabajo en línea (MS Teams, Zoom, entre otros).

- Soporte en otras herramientas anteriormente disponibles, pero no tan utilizadas (exámenes en línea, y otras funcionalidades del aula virtual, por ejemplo).

- Diseño y soporte en la organización de las defensas de Trabajos de Fin de Grado (todas en línea).

- Aumento de las asistencias personalizadas (por correo electrónico, teléfono y videollamada).

- Soporte en tiempo real de los exámenes de fin de semestre (todos en línea). Guardias organizadas con canal de contacto por MS Teams.

- Coordinación con otras áreas para tratar temas transversales, debido al aumento de la digitalización (Área de las TIC, Biblioteca, servicios generales, secretarías de centro, entre otras).

El inicio del curso 2020-2021 se planteó con una presencialidad reducida, pero duró poco más de un mes. A partir de mediados de octubre se limitó la presencialidad a las partes prácticas de algunas asignaturas.

$\mathrm{Al}$ inicio del segundo período, se tomaron las siguientes decisiones:

- Ampliar y reorganizar la unidad.

- Ofrecer un sistema de videoconferencia para las sesiones en línea, integrado con el aula virtual y que permitiera autonomía al profesorado ${ }^{1}$

\footnotetext{
${ }^{1}$ En septiembre de 2020 se optó por BigBlueButton, una propuesta con muy buena integración con Moodle, la plataforma de aprendizaje de la universidad. A partir de finales de noviembre, se implantó el uso de Zoom con licencia campus para todo el personal de la UVic-UCC.
} 
- Proveer a las facultades de aulas híbridas, preparadas para impartir docencia con alumnos presenciales y en línea a la vez.

- Ampliar la oferta de formación (ofrecida desde el Centro de Innovación y Formación en Educación) y disminuir (pero mantener) las sesiones de asesoramiento de tema libre.

- Potenciar el sistema URespon para gestionar las ayudas concretas al profesorado.

- Elaborar y publicar materiales de soporte según las solicitudes de ayuda recibidas por parte del profesorado.

- Preparar a la comunidad para el uso de una herramienta de portafolios (Mahara) para dar más versatilidad a los trabajos de los estudiantes.

- Destinar personal de soporte propio para la Facultad de Medicina.

\section{Resultados}

\subsection{Sesiones de formación}

Entre el primer y el segundo período se han realizado 60 sesiones en línea de soporte al profesorado.

Durante el primer período, se plantearon dos veces a la semana, alternando día y franja horaria. De las 37 realizadas, 25 fueron genéricas (con los temas determinados por los asistentes) y 12 fueron temáticas (cuestionarios, defensas de TFG, gestión de reuniones, gestión de vídeos). Algunas de estas sesiones se orientaron específicamente al Personal de Administración y Servicio (PAS) que dan soporte en el ámbito de la docencia.

En el segundo período, se han realizado 23 sesiones. La frecuencia de las genéricas ha disminuido (cada quince días) y se ha aumentado las sesiones de formación específicas, que han sido 19 (cuestionarios, sistemas de videoconferencias, evaluación, rúbricas, sesiones de iniciación para profesorado de nueva incorporación, H5P para elaborar material docente interactivo, gestión de equipos).

Todas estas sesiones han sido ofrecidas y certificadas desde el Centro de Innovación y Formación en Educación (CIFE: unidad responsable de la formación interna del profesorado de la universidad, entre otras funciones).

En la Figura 1 se muestra la asistencia de cada sesión. Se observa que las sesiones con temáticas que tienen lugar en el momento en el que el profesorado necesita esta información tienen una asistencia muy elevada. Las más concurridas en el primer período trataron temas de cuestionarios en línea (91, 65 y 62 asistentes), gestión de reuniones/clases en línea (76) y gestión de vídeos (52). En el segundo período, las sesiones con más participación se centraron en sistemas de videoconferencia para impartir docencia en línea $(114,100$, 86 y 68) y el sistema de detección del plagio (58). 


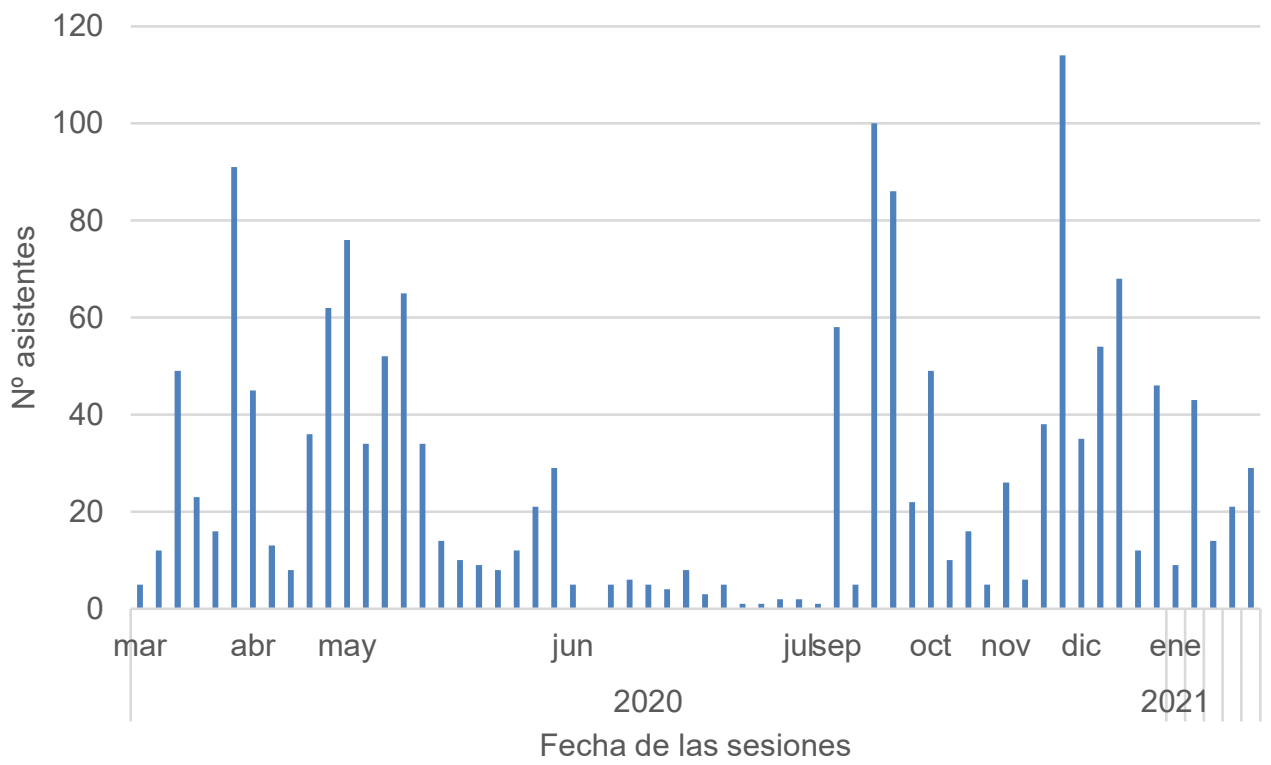

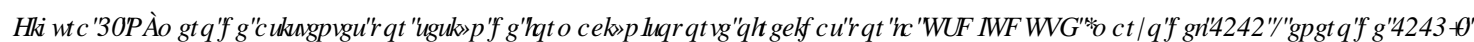

Si nos fijamos en el porcentaje de certificados obtenidos (respecto a los asistentes inscritos) en las sesiones de formación correspondientes al 2021 (con inscripción y certificadas por el CIFE), en la mayoría de los casos con ejercicio autónomo posterior obligatorio, los resultados (Figura 2) indican que hay temas que generan más interés. Estos temas tratan las herramientas de videoconferencia (BigBlueButton), la detección del plagio (Urkund), y el apoyo de PAS a PDI, principalmente en tareas de gestión de videoconferencia. De hecho, una vez más se nota que las videoconferencias, o sea, las sesiones sincrónicas en línea son la máxima prioridad de los asistentes.

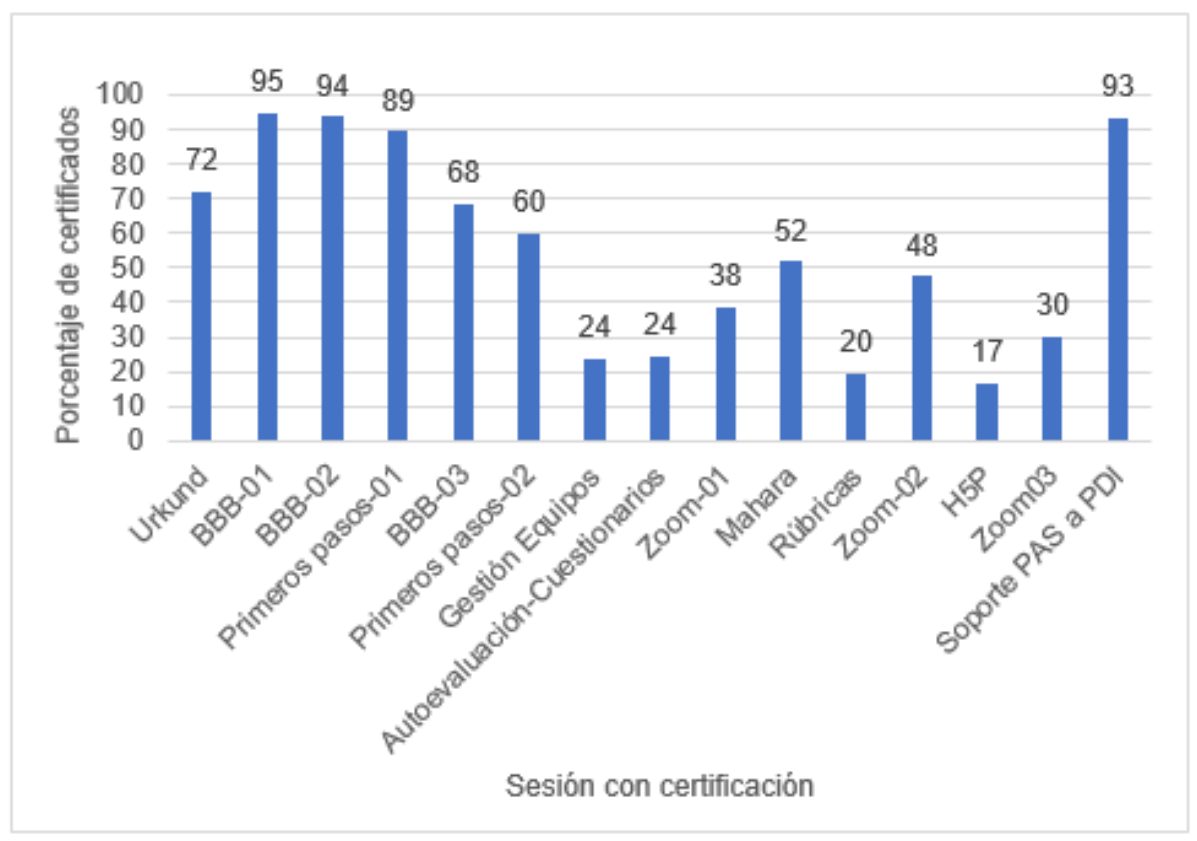

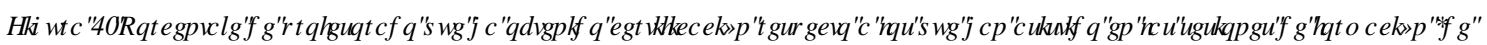

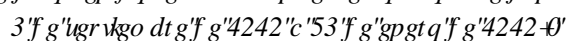


La satisfacción media global de estas formaciones es de 3,51 sobre 4 (con una participación promedio de $39,8 \%$ ) (Figura 3).

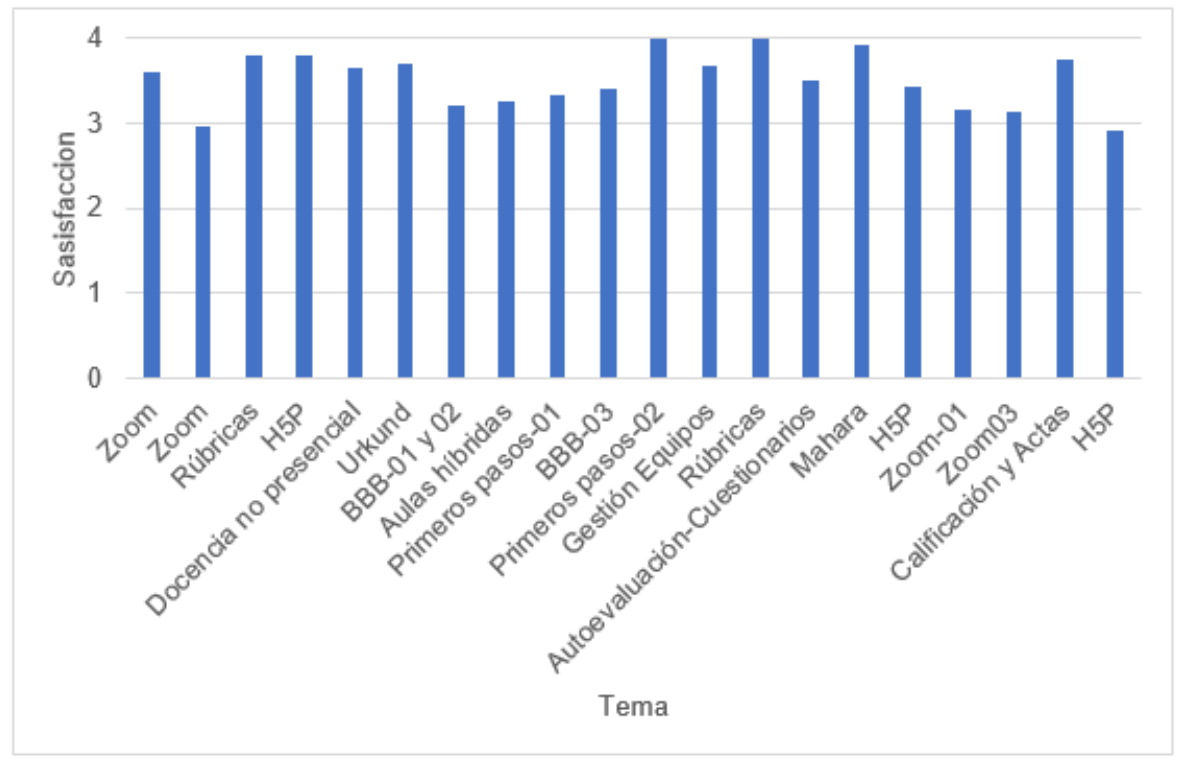

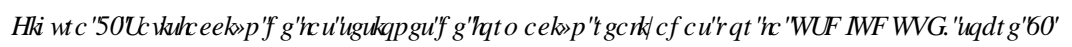

\subsection{Material de soporte}

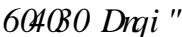

Desde el 2014, el blog de la UDUTE (mon.uvic.cat/udute) se ha usado como plataforma para la publicación de páginas de ayuda y adaptación a la situación derivada de la COVID con páginas de información fija:

- Acciones básicas en la docencia no presencial (12 de marzo)

Se trata de una página breve, con explicaciones y clips de vídeo para mostrar los aspectos más importantes de la docencia no presencial.

- Hacia la docencia no presencial. Las alternativas en línea (12 de marzo)

Es una página más detallada sobre las alternativas no presenciales a diversas actividades presenciales.

- Coronavirus. Apoyo para la docencia no presencial (12 de marzo)

Página que detalla las iniciativas y actividades de soporte que se organizan para enfrentar la situación de confinamiento y docencia no presencial.

- Apuntes sobre la docencia no presencial (VOA) (16 de marzo)

Es una página complementaria con más consejos académicos.

- Orientaciones para la evaluación en línea (25 de abril)

Indicaciones institucionales y buenas prácticas.

Desde el principio del confinamiento el ritmo de publicaciones ha aumentado respecto a los años anteriores, concentrándose en la primera parte del primer período (más de 10 entradas nuevas por mes) (Figura 4). 


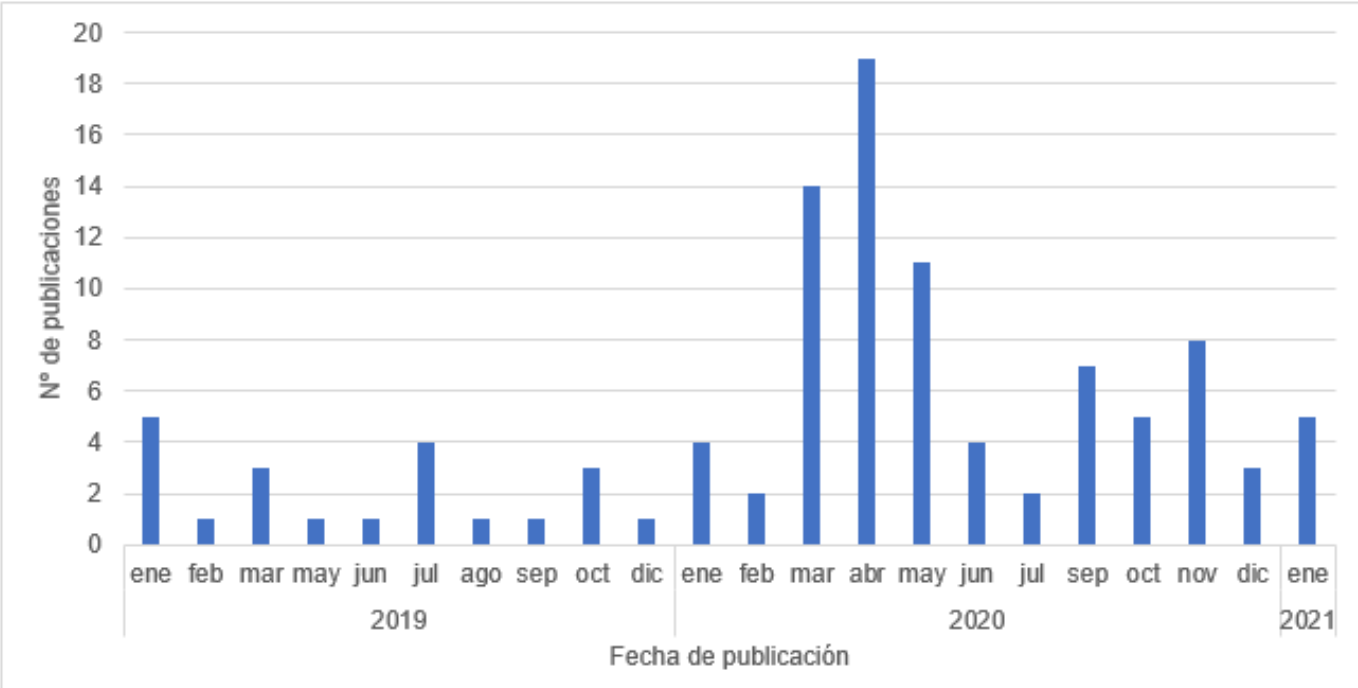

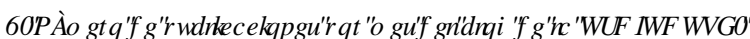

) $L X W$

Si revisamos el número de visitas recibidas, observamos el aumento considerable a partir del inicio del confinamiento en marzo de 2020 (de un promedio de menos de 1.000 visitas mensuales a más de 6.000 en el primer período y entre 3.000 y 5.000 en el segundo período) (Figura 5).

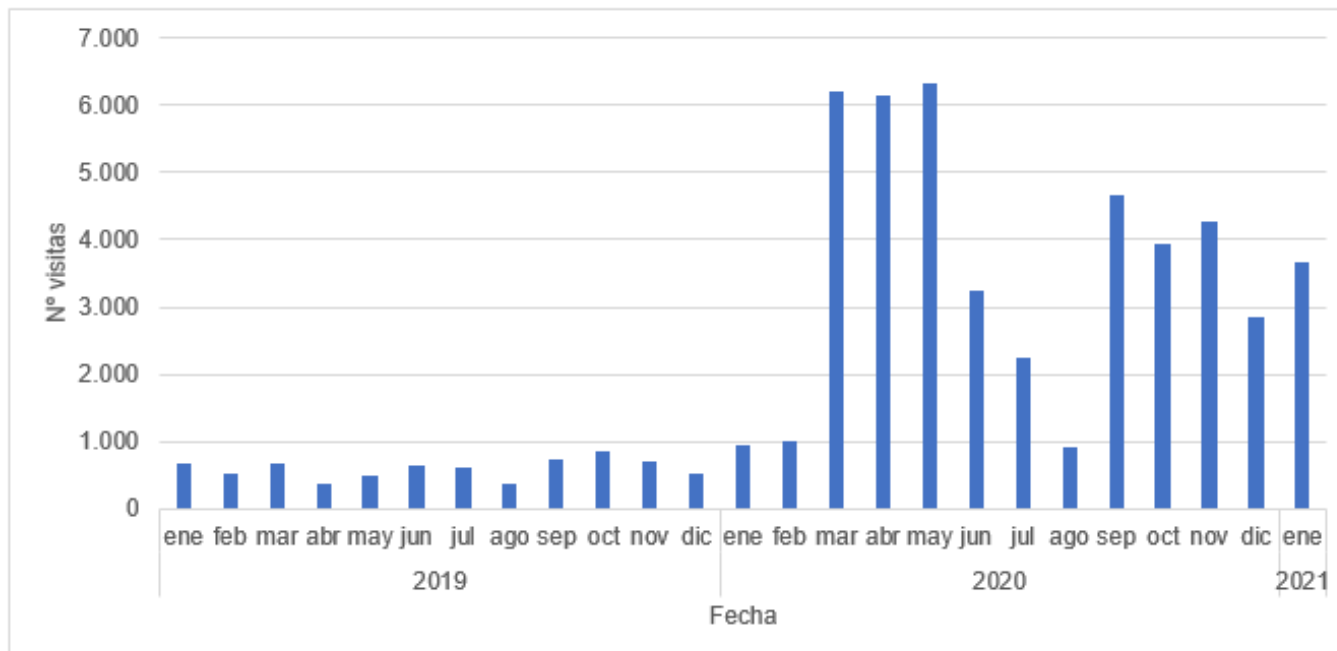

) $I J X W$

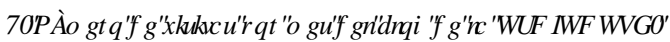

Desde febrero de 2020 a enero de 2021, las páginas con más de 100 visitas han sido las que se muestran en la Tabla 1 (con el número de visitas acumuladas). 17 de las 20 más visitadas han sido publicadas de marzo de 2020 a enero de 2021.

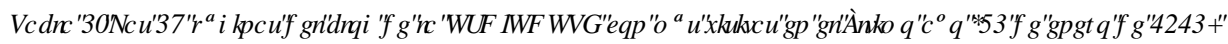

\begin{tabular}{clc}
\hline $\mathbf{N}^{\mathbf{0}}$ & Página & Número de visitas \\
\hline 1 & Home page / Archives & 8.504 \\
\hline 2 & Las calificaciones en Moodle & 3.531 \\
\hline 3 & Formato Aiken para crear rápidamente las preguntas de tipo test & 2.734 \\
\hline 4 & Coronavirus. Soporte para la docencia no presencial & 2.484 \\
\hline 5 & Reuniones Zoom. Más detalles de acceso y configuración & 1.865 \\
\hline
\end{tabular}

(c)) BY-NC-ND 2021, Universitat Politècnica de València

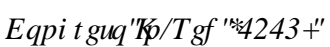




\begin{tabular}{clc}
\hline 6 & Apuntes sobre la docencia no presencial (VOA) & 1.177 \\
\hline 7 & Grabación de clips de vídeo en un ordenador portátil & 1.057 \\
\hline 8 & Hacia la docencia no presencial. Las alternativas en línea & 1.010 \\
\hline 9 & El Calificador de Moodle (otra vez) & 999 \\
\hline 10 & Recomanaciones para usar cuestionarios de Moodle (no presencial) & 953 \\
\hline 11 & Como configurar una actividad BigBlueButton & 829 \\
\hline 12 & Acciones básicas para la docencia no presencial & 796 \\
\hline 13 & La actividad Zoom en Moodle & 744 \\
\hline 14 & Grabación de vídeos en casa. Escenografía y recursos & 731 \\
\hline 15 & Plataformas de videoconferencia durante el curso 2020-2021 & 640 \\
\hline
\end{tabular}

\section{१एपि * UEDFIRQHMGHYTGH]}

La USD/UDUTE dispone desde el 2016 de un espacio en YouTube donde publica en oculto los videotutoriales y las grabaciones de las sesiones de formación. El canal se usa también para otro tipo de vídeos. En la Figura 6 se muestra el número de vídeos publicados, que ha aumentado sobre todo en la primera parte del primer período (más de 10 videotutoriales los meses de marzo a mayo de 2020). Después del primer período, la mayoría de los vídeos publicados han sido de sesiones de formación/soporte.

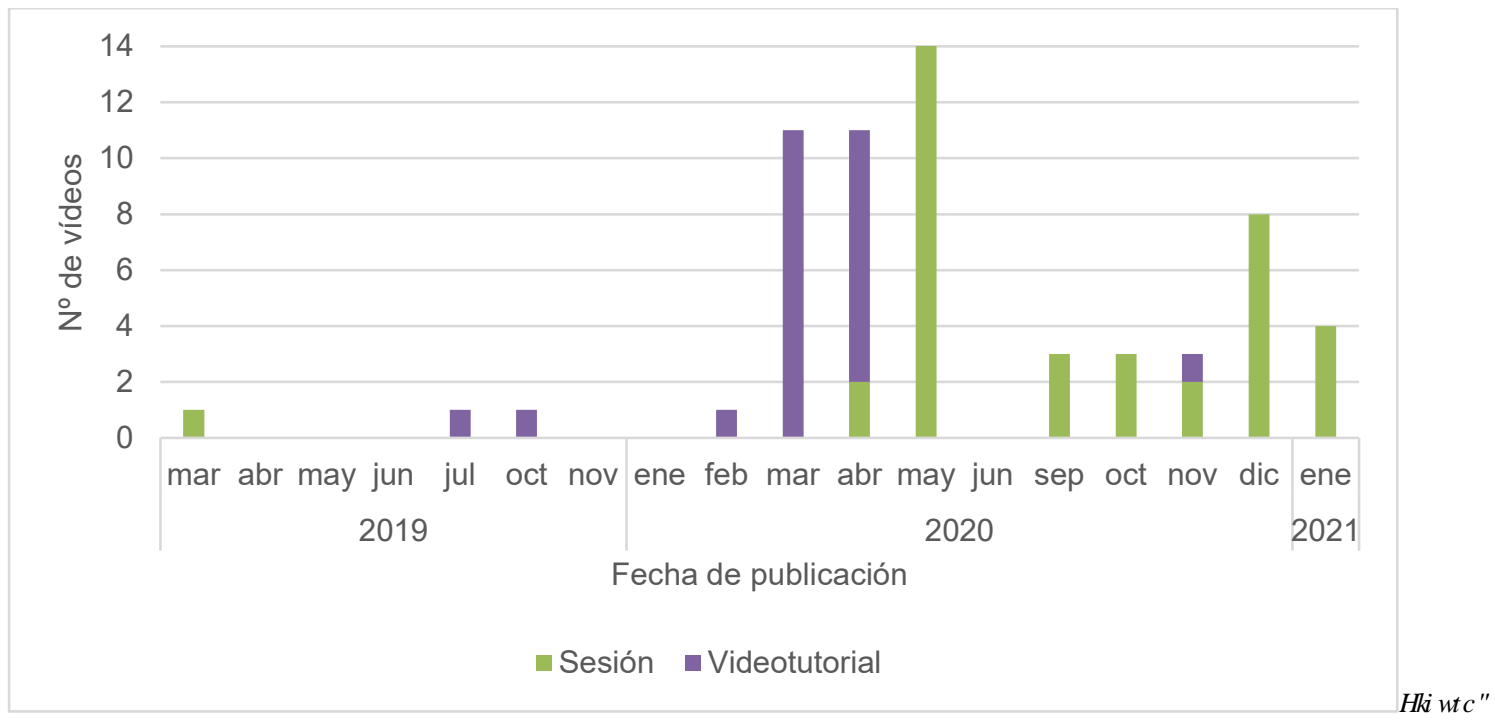

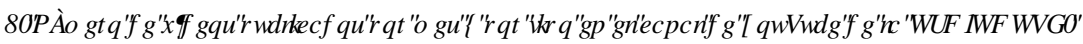

En la Figura 7 se muestran las visitas por mes de los vídeos publicados en el canal de la USD/UDUTE. En el primer mes de confinamiento aumentaron de menos de 100 a casi 800 . Fue decreciendo en los siguientes meses y parece estabilizada alrededor de las 200 .

En la Tabla 2 se muestran los videos de sesiones y videotutoriales más visualizados, con el número total de visualizaciones desde el inicio del canal hasta 31 de enero del 2021. 13 de los 20 más vistos fueron publicados entre marzo del $2020 \mathrm{y}$ enero de 2021. 


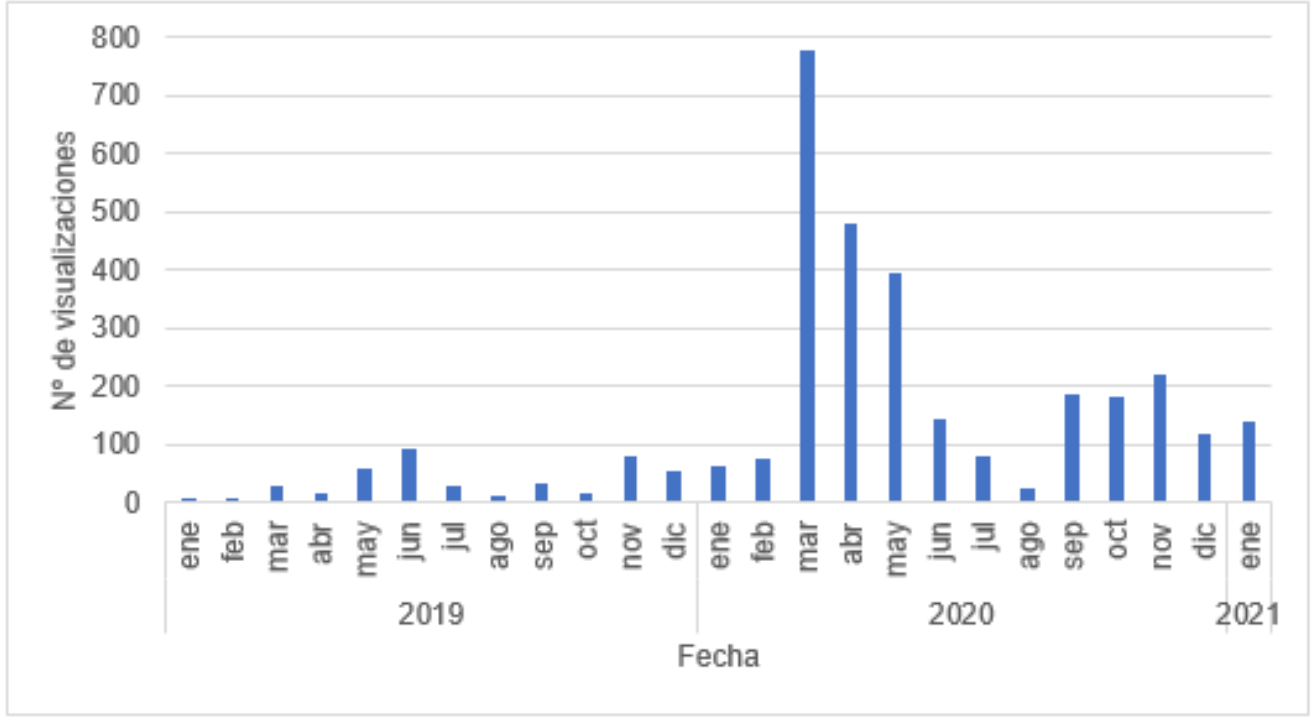

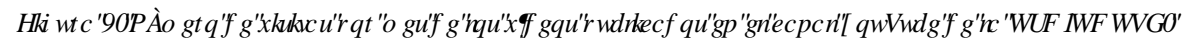

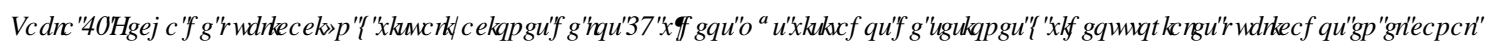

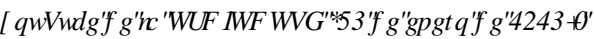

\begin{tabular}{clcc}
\hline $\mathbf{N}^{\mathbf{2}}$ & \multicolumn{1}{c}{ Vídeo } & $\begin{array}{c}\text { Fecha } \\
\text { publicación }\end{array}$ & Visualizaciones \\
\hline 1 & Videollamadas con Microsoft Teams & 12 -mar-20 & 403 \\
\hline 2 & Rúbrica en una tarea de Moodle & 01 -jul-19 & 265 \\
\hline 3 & Grabar material docente con MS Teams & 20 -mar-20 & 211 \\
\hline 4 & 1. Presentación de las aulas virtuales & 09 -nov-16 & 205 \\
\hline 5 & Resultado del análisis en sistema Urkund & 15 -feb-17 & 186 \\
\hline 6 & Foro de avisos y noticies: tablón del profesorado & 11 -mar-20 & 173 \\
\hline 7 & Aulas virtuales 2017 -2018 & 05 -sep-17 & 153 \\
\hline 8 & Revisar los resultados de un cuestionario de Moodle & $14-$-abr-20 & 144 \\
\hline 9 & Revisión de la tarea-Sistema de plagio Urkund & 08 -feb-17 & 141 \\
\hline 10 & Banco de preguntas de Moodle & 02 -abr-20 & 123 \\
\hline 11 & OBS Studio & $07-$ may-20 & 123 \\
\hline 12 & Foro general de la asignatura & 11 -mar-20 & 112 \\
\hline 13 & Restaurar un aula 2017-2018 & 05 -sep-17 & 105 \\
\hline 14 & Utilizar un aula Moodle como estudiante & 17 -abr-20 & 94 \\
\hline 15 & 2. Entrada al Campus Virtual y acceso a las aulas & 09 -nov-16 & 93 \\
\hline
\end{tabular}

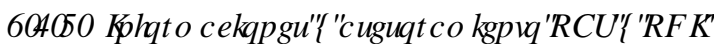

Es un aula Moodle donde se publican los documentos elaborados conjuntamente por el Vicerrectorado de Ordenación Académica y las prefecturas de estudios de los diferentes centros.

- Evaluación en el contexto de no presencialidad (COVID-19) (25 de abril 2020, posteriormente actualizado en diciembre de 2020). (D1)

- Criterios de aplicación en la docencia virtual (indicaciones de uso de cámaras y grabaciones en la adaptación de la presencialidad a la virtualidad). (D2) 
- Indicaciones para el primer semestre 2020-2021 (julio de 2020). Estrategias para la planificación de la docencia. (D3)

- Evaluación no presencial. Orientaciones y recursos para llevar a cabo con éxito la evaluación no presencial en el contexto del período excepcional COVID-19. (D4)

- Guía del estudiante 2020-2021. Buenas prácticas en la redacción de contenidos para la guía del estudiante (julio de 2020). (D5)

- Repositorio de recursos para la docencia (wiki en edición). (D6)

También se usa como sistema de comunicación de las novedades relacionadas con todos los temas que se tratan en este artículo. En el foro de profesorado los mensajes quedan ordenados y se pueden hacer búsquedas. Com archivo es muy superior al correo electrónico y quita barreras al conocimiento de profesorado de nueva incorporación.

Los participantes de esta aula están sincronizados con los miembros de la comunidad PAS y PDI, de manera que no hay que pensar en actualizaciones por modificaciones de contrataciones.

Algunos de estos documentos se han compartido por la comunidad también a través de otros sistemas (correo electrónico, espacio propio de las facultades). Las visitas registradas a estos recursos se muestran en la Figura 8. Para ello se han eliminado las visitas repetidas el mismo día del mismo recurso y usuario.

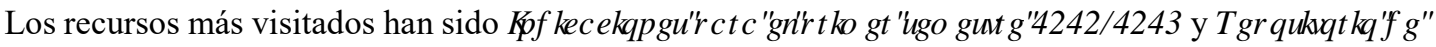
UFXURVSDUDUCRFHED. Este segundo está en proceso de revisión y por eso no se ha potenciado su uso.

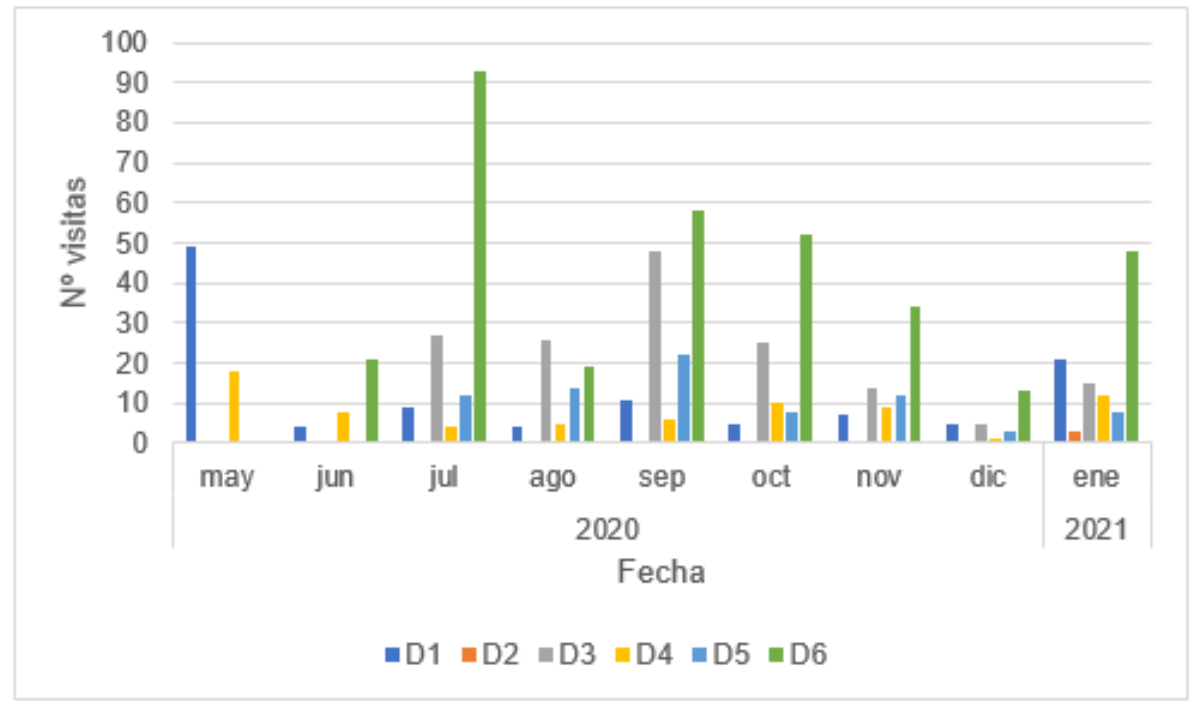

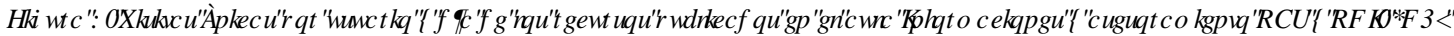

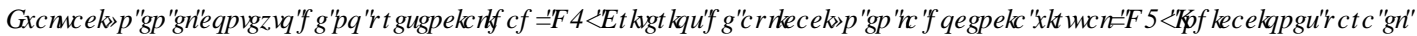

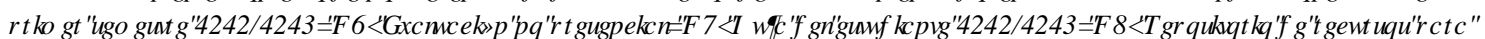
(D) CRFHQFDW

प

\subsection{URespon}

URespon es una herramienta de petición de servicio que tiene la universidad para dar soporte a diferentes temas a partir de solicitudes enviadas por formulario. La USD/UDUTE dispone de un canal URespon 
propio para que los profesores hagan sus consultas a través de un formulario que incluye la fecha, la descripción, el tema (con la opción de escoger de una lista), entre otros campos. Todo el personal que gestiona las peticiones puede ver la consulta, asignársela para gestionarla, escribir mensajes de seguimiento y adjuntar información e imágenes para responder.

Este servicio se puso en marcha al inicio del confinamiento y permitió amplificar la respuesta de la USD/UDUTE ya que podía ser atendido de manera más ordenada y ágil que por correo electrónico, teléfono y asistencia personalizada presencial (que eran los canales más usados hasta ese momento). Se atendieron 395 solicitudes en el primer período y 844 en el segundo.

Durante el segundo período se dio de alta un formulario interno para poder registrar las asistencias que no se gestionaron directamente a través del URespon (correo electrónico, teléfono, videollamada...). Entre el 20 y el $40 \%$ de las asistencias se hacen aún en este formato (Figura 9).

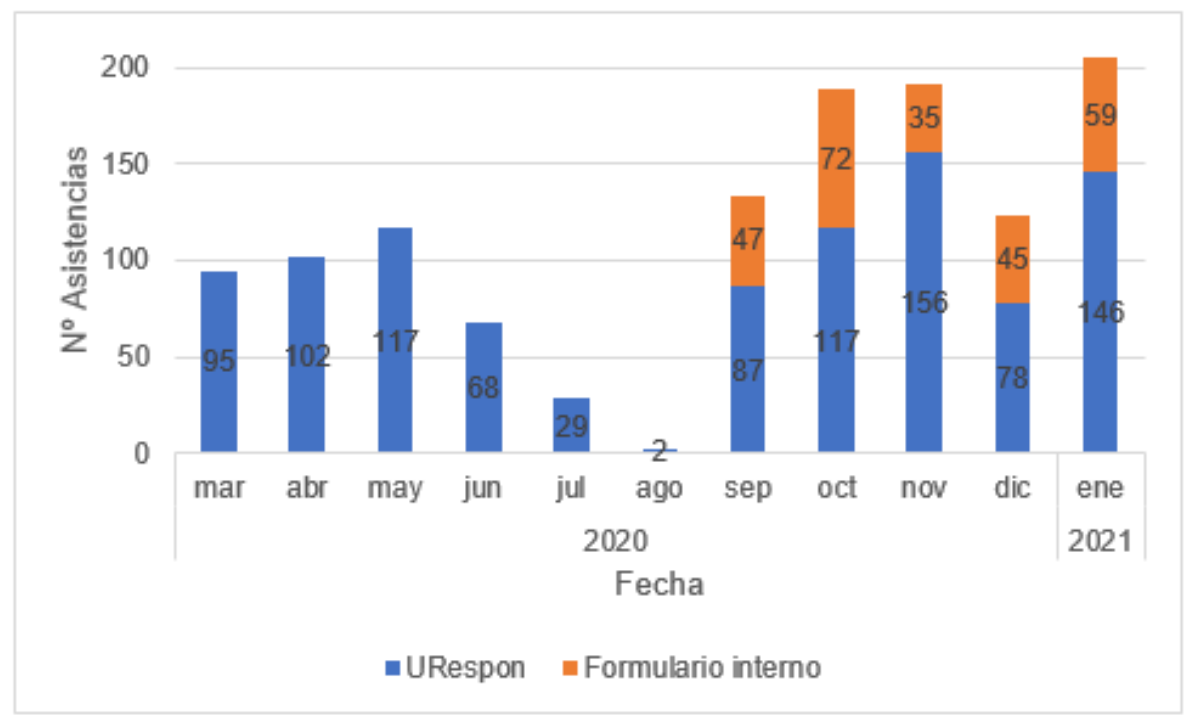

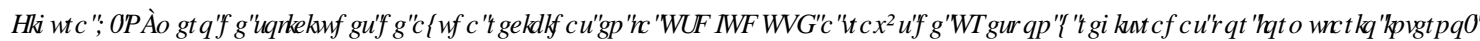

La mediana mensual de la satisfacción de estas asistencias oscila entre el 4,56 y el 4,93 sobre 5 (Figura 10).

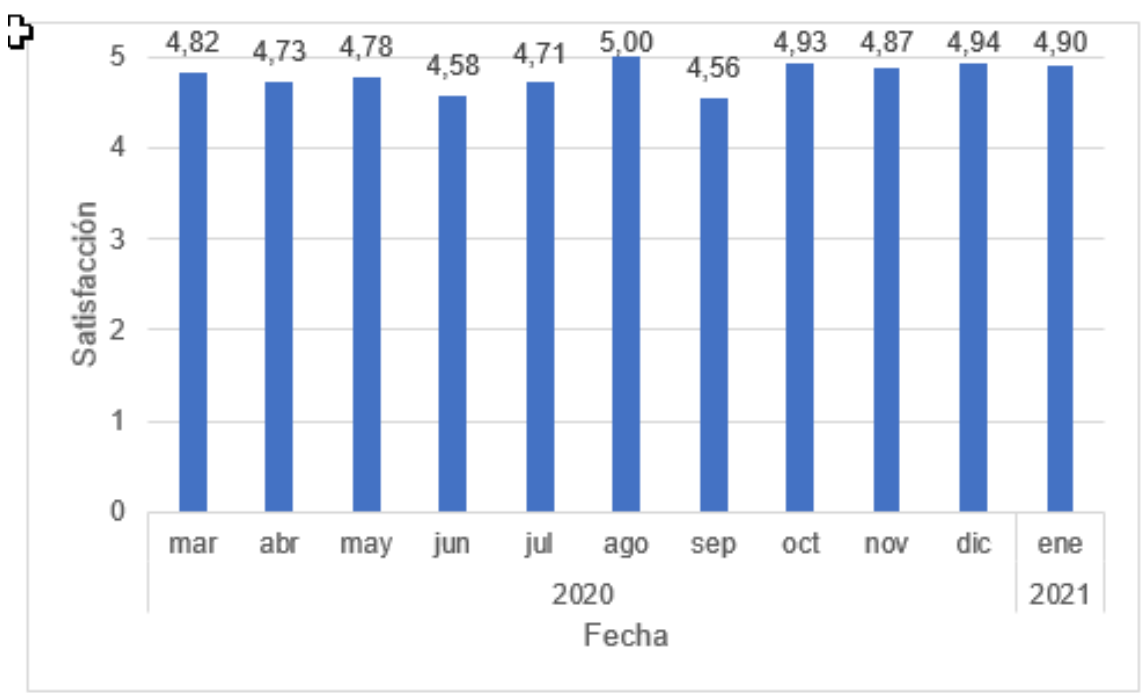

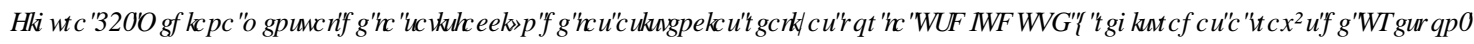




\section{Conclusiones}

De las acciones llevadas a cabo podemos concluir:

\subsection{Sesiones/cursos de formación}

- Ha sido una estrategia con una satisfacción elevada por parte del profesorado, por lo que se considera útil seguir con ella.

- En algunos casos la valoración ha sido algo irregular, probablemente por la dificultad de ajustar un nivel adecuado para todos los asistentes.

- El contexto del momento académico y de los cambios a realizar determinan el éxito en la asistencia de las formaciones. Probablemente debido al trepidante ritmo de las adaptaciones necesarias, el profesorado asiste a las sesiones si en aquel momento necesita ese conocimiento/experiencia.

- La obtención de certificación no siempre es una prioridad por parte de los asistentes.

\subsection{Material de soporte}

पषा० \%बग

- El número de visitas ha aumentado mucho, con valores más altos durante el primer período observado, por lo que se considera una herramienta útil para informar/promover conocimiento.

- Los contenidos más consultados identifican temas “estrella", que són más necesarios para el profesorado, y no siempre los más recientes.

- Se valora la necesidad de diversificar la lengua de redacción, para dar soporte a un rango más grande del profesorado.

- Es mejor concentrar esfuerzos en elaborar contenido que aporte valor añadido (especificidades de la universidad, usos concretos de las herramientas, por ejemplo, que redactar información más general que ya está escrita y es posible enlazar.

\section{१०१० * UEDFlyQGHYtGRV}

- El número de visitas ha aumentado mucho, sobre todo en el primer período, que es cuando se elaboraron más videotutoriales, por lo que hay que potenciar la elaboración de vídeos que expliquen acciones concretas.

- Los contenidos más consultados identifican temas "estrella", que son más necesarios para el profesorado

- En general las sesiones de formación no son visualizadas a posteriori, a pesar de ello, se considera importante seguir con su publicación.

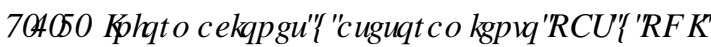

- Las visitas recibidas son más elevadas para aquellos documentos que no han sido compartidos por otros medios con la comunidad.

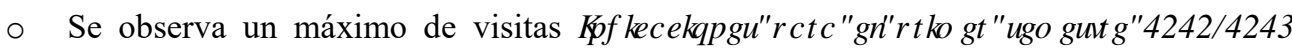
durante el período en que el profesorado concreta la planificación para el siguiente curso.

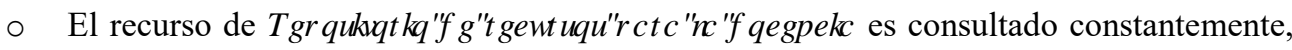
por lo que hay que optimizarlo y promocionarlo entre el profesorado. 


\subsection{URespon}

- El nivel de satisfacción de este tipo de atención es muy alto. Se interpreta que es por la rapidez y la gran posibilidad de personalización: a menudo el profesorado prefiere preguntar a buscar la respuesta en los diferentes entornos que tiene disponibles.

- A pesar del buen resultado del formulario URespon, no disminuyen las solicitudes fuera del formulario, que comportan interrupciones en el día a día (por teléfono, correo electrónico y videollamada) y ocupan más tiempo al equipo que responde. Deberíamos poder disminuir el porcentaje de las consultas por estas vías.

- Habría que hacer una reflexión sobre las preguntas más repetidas y reutilizar las respuestas, a la vez que se puede elaborar material de soporte que ayudaría a responder con más fluidez.

\subsection{Una reflexión final}

La pandemia COVID-19 y los consiguientes períodos de confinamiento han obligado a la comunidad universitaria a aprender nuevas formas no presenciales de mantener los programas formativos existentes, a pesar de las reticencias de profesorado y estudiantes. El contexto de necesidad imperante ha permitido dar prioridad a redimensionar la unidad de apoyo UDUTE, dotándola de más recursos humanos y capacidad de intervención. A la vez se ha sistematizado el servicio para poder sacar más rendimiento y poder analizar su uso. Los datos indican que el profesorado acude cada vez más a este servicio para intentar resolver sus problemas docentes y en este sentido se ha creado una dinámica de petición / respuesta que atiende los desafíos emergentes.

Sea como sea, las prioridades de muchos se han centrado naturalmente en poder seguir con el modelo de sesiones de clase sincrónica, ahora por videoconferencia, en las cuales el protagonismo es del equipo docente, y la gestión de pruebas en línea para sustituir los exámenes presenciales. En este sentido, el aprovechamiento de la crisis sanitaria para fomentar la innovación pedagógica es menor, ya que la creación de recursos y actividades que los estudiantes pueden explotar de forma asincrónica -el plato fuerte de la docencia con recursos de tecnología educativa- requiere análisis, tiempo y reflexión. Algo se ha podido hacer, pero la urgencia del momento no ha permitido preparar respuestas más elaboradas.

Ahora que nos acerquemos al período post-pandemia, muchos se preguntan qué quedará de las nuevas técnicas y metodologías docentes. Es evidente que no volveremos al estadio de antes y se harán más actividades en línea, en contextos en los cuales antes era imposible, por las razones que sean. Si solo es por la flexibilización de actitudes resistentes al cambio, con una gestión adecuada, el acervo de la pandemia y su efecto trampolín podrían ser considerables.

\section{Referencias}

ALEXANDER, B., ASHFORD-ROWE, K., BARAJAS-MURPHY, N., DOBBIN, G., KNOTT, J., MCCORMACK,

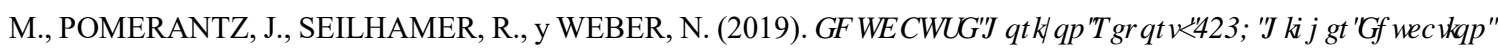
( GIURQ Louisville, CO: EDUCAUSE, 2019.

ADAMS BECKER, S., CUMMINS, M., DAVIS, A. FREEMAN, A., HALL GIESINGER, C. y

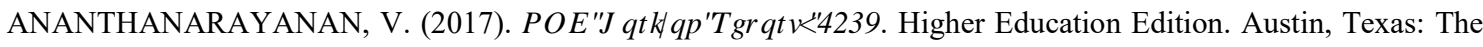
New Media Consortium, 2017.

ADELL, J., I CASTAÑEDA, L. (2010). "Los entornos personales de aprendizaje (ples): Una nueva manera de entender

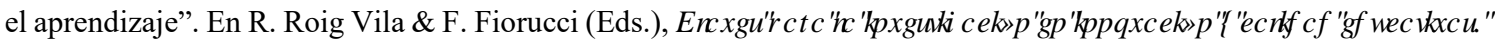

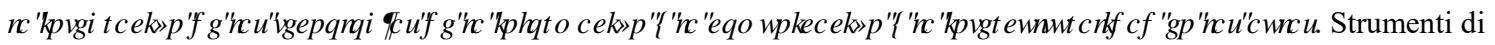
recerca per l'innovazione e la qualità in ambito educativo, le tecnologie dell'informazione e della comunicazione e l'interculturalità nella scuola. Alcoy-Roma: Marfil--Roma TRE Universita degli Studi. 
ÁREA-MOREIRA, M., HERNÁNDEZ-RIVERO, V. y SOSA-ALONSO, J.J. (2016). "Modelos de integración didáctica de las TIC en el aula" en \&RP XQQFDU 47(24), 79-87.

BROWN, M., MCCORMACK, M., REEVES, J., BROOKS, D. C., y GRAJEK, S. (2020). पिएव( ' $8 \& \$ 8$ 6( प+RU]RQ 5 HSRUW7 HEFIQJ DDQGV HDQTQIL GIMRQ(Louisville, CO: EDUCAUSE, 2020).

COLL, C. (2004). "Psicología de la educación y prácticas educativas mediadas por las tecnologías de la información y la comunicación” en 6IQpFUFD, 25, 1-24.

COLL, C., MAURI, T. y ONRUBIA, J. (2008). "Análisis de los usos reales de las TIC en contextos educativos formales: una aproximación sociocultural” en 5HIWD( GFWU QIFDGH, QYHWWDFIyQ( GXFDUYD, 10 (1). Recuperado en http://redie.uabc.mx/vol10nol/contenido-coll2.html

CÓRICA, J. L. (2020). Resistencia docente al cambio: Caracterización y estrategias para un problema no resuelto.

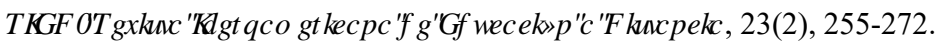

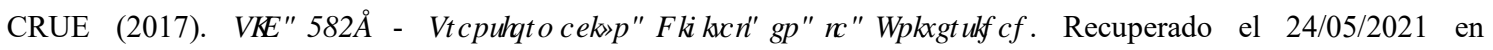
https://tic.crue.org/wp-content/uploads/2016/03/transformacion-digital-univ.pdf

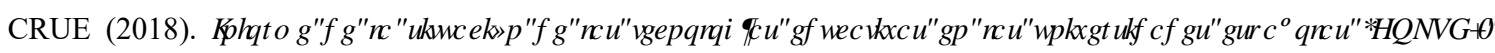
Recuperado el 24/05/2021 en! https://tic.crue.org/wp-content/uploads/2020/09/Tecnologias-Educativas2018 DIGITAL.pdf

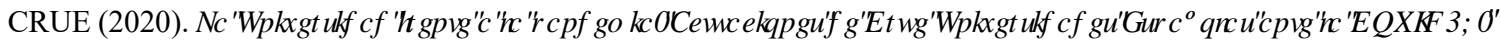
Recuperado el 24/05/2021 en https:/www.crue.org/wp-content/uploads/2020/12/La-Universidad-frente-a-laPandemia.pdf]

CUEN MICHEL, C., RAMÍREZ ROMERO, J. L. (2013). "Usos, funciones y efectos de las TIC en el aprendizaje de una licenciatura en Ciencias de la Comunicación" en ( ' 87 ( \& Costa Rica 2013.

ESTEVE, F. (2009). "Bolonia y las TIC: de la docencia 1.0 al aprendizaje 2.0” en / D\&\&XHUy Q8 QIYHULDUD, 5, 58-67.

GISBERT, M. (2017). "La digitalización de las universidades”en 7HFQROtDHQD DHFDD vol. 17 № 3 Especial.

GROS, B. (2016). "Retos y tendencias sobre el futuro de la investigación acerca del aprendizaje con tecnologías digitales" en 5( ' П5 HILWDGH( GXFDFIy QDD' LWDDFD. 50(10). Recuperado en http://www.um.es/ead/red/50

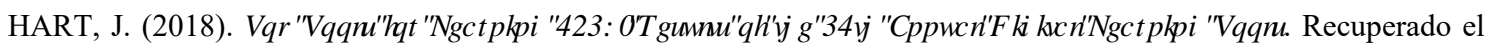
22/11/2018 en https://www.toptools4learning.com/

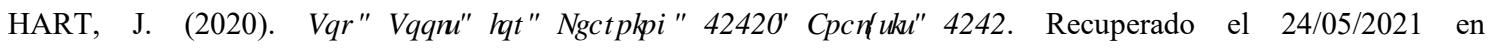
https://www.toptools4learning.com/analysis-2020/

MERCADER, C. y GAIRÍN, J. (2017). “¿Cómo utiliza el profesorado universitario las tecnologías digitales en sus aulas?” en 5( ' 8 प5 HILWDGH' RFHQFD8 QIYHUWDUD, 15(2), 257-274. https://doi.org/10.4995/redu.2017.7635

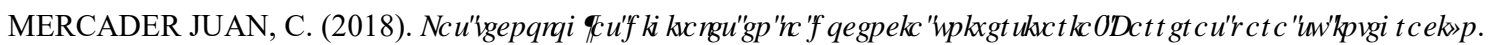

Tesis doctoral. http://hdl.handle.net/10803/662771

PRIESCA, P. (2016). I D WDQMRP DFIYQ GJIWD GH DV HPSUHDV. Recuperado el 10 de octubre de 2018 en https://www.fundacionctic.org/ctic/articulos-y-otras-publicaciones/la-transformacion-digital-de-las-empresas

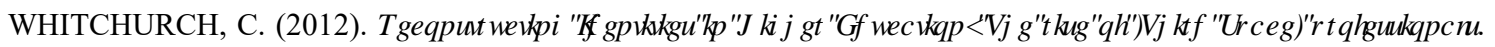
New York: Taylor and Francis.

ZEMPOALTECA, B., BARRAGÁN, J.F., GONZÁLEZ, J. y GUZMÁN, T. (2017). “Formación en TIC y competencia digital en la docencia en instituciones públicas de educación superior" en \$SHUWD, 9(1), 80-96.

(cc) EY-NC-ND 2021, Universitat Politècnica de València

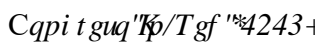

